\title{
La Cuarta revolución industrial: Un paradigma emergente en la sociedad de hoy
}

\section{doi: $10.52749 /$ fh.v2i4.3}

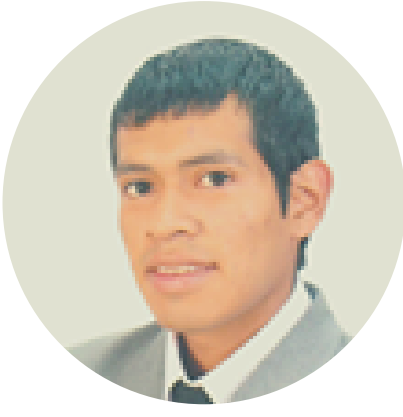

\section{WILDO SIANCAS}

Psicólogo por la Universidad San Ignacio de Loyola. Cuenta con estudios de maestría en Educación con mención en Psicología educativa. Se ha desempeñado como docente en organizaciones no gubernamentales orientadas al sector educativo y en psicología clínica infantil. Consultor independiente en metodología de la investigación. Miembro de la Sociedad Secular Humanista del Perú.

wildo_esmith_14@hotmail.com

Resumen. Las revoluciones industriales se han caracterizado por mantener un auge y marcar una tendencia a lo largo del proceso histórico-cultural de la humanidad. Esto se debe a que el avance científico y tecnológico es una realidad innegable que contribuye con el desarrollo humano. Como resultado, los avances tecnológicos han traído consigo un marcado progreso exponencial, tanto en conocimiento, como en problemáticas de tipo social y económico. Esto se debe a que los estados y las revoluciones involucran ciertas manifestaciones previas, tales como: El crecimiento asimétrico de los recursos, la diferenciación en todos los estratos y la generación de un sistema nuevo dentro del actual. Razón por la cual, una formación científica y ética requiere una cultura consolidada bajo una perspectiva humanista. Para ello, el presente proyecto consiste en una investigación teórica, enmarcada en los hitos de la revisión narrativa-conceptual, acerca de aquel constructo definido como Cuarta revolución industrial. Asimismo, se han de examinar los principales impactos de este paradigma emergente en las dimensiones biologicas, psicológicas, sociales y contextuales de la sociedad.

Palabras clave: biología, contexto, Cuarta Revolución Industrial, psicología, sociedad

\section{Introducción}

Las revoluciones se han caracterizado por mantener un auge y marcar una tendencia a lo largo del proceso histórico-cultural del ser humano. Una de ellas, la Cuarta Revolución industrial, es definida como "el conjunto de cambios o transformaciones radicales en la vida social humana que ha sido provocado por la aparición, implementación y uso de una nueva generación de tecnologías" (Gayozzo, 2020). Sibien fueron tres etapas industriales por las cuales la humanidad ha atravesado, su progreso, en vez de ser disruptivo, fue paulatino y acorde al contexto histórico-cultural de la época en que surgieron (Torró, 1999). La primera etapa involucró la mecanización de la producción mediante la energía a vapor; la siguiente se caracterizó por el uso de la energía eléctrica para incrementar la producción en masa. Del mismo modo, el tercer proceso industrial, trajo consigo la automatización de los bienes y servicios a través de la electrónica aplicada a las tecnologías de la información. De ahí que el carácter acumulativo de aquellos procesos exponenciales permita el surgimiento de nuevas nuevas formas de aplicar las tecnologías NBIC (Nanotecnología, Biotecnología, Tecnologías de la información y Ciencias cognitivas) en el futuro desarrollo del contexto humano. Ante ello, la Cuarta revolución industrial, también denominada Industria 4.0 o bajo las siglas $4 R$, es un término cuya idea se plantea como "un fenómeno social motivado por un conjunto de tecnologías emergentes y avanzadas que permiten la fusión de los mundos físico, digital y biológico" (Schwab, 2016). En otros términos, la integración interdisciplinaria entre diversas áreas de las ciencias, trae consigo resultados exponenciales que permiten un mayor progreso de la sociedad y sus futuras generaciones. Este paradigma se caracteriza por el aumento de la velocidad y la amplitud exponencial del impacto mediante la integración transdisciplinaria de las diversas áreas del conocimiento. Ello con el fin de establecer principales puntos de inflexión en el desarrollo del avance científico y tecnológico dentro de un marco de crecimiento constante. Entre los principales hitos, Gayozzo (2020) define cinco áreas de desarrollo que manifiestan el carácter innovador 
de este paradigma emergente. Éstas suelen abarcar megatendencias tales como la automatización de procesos mediante el Machine learning, la manipulación genética de seres vivos y la creación de nuevos materiales a escalas manométricas. Asimismo, el autor también refiere a la manufactura de productos y servicios interconectados en la nube, así como el auge de ecosistemas físico-digitales, como indicadores relevantes que forman parte del auge de la Cuarta revolución industrial. Aspectos que han marcado puntos de inflexión exponencial en la dimensión Biológica, Psicológica, Social y Contextual del ser humano. Cada una de ellas con sus inherentes alcances en cuanto a su propia naturaleza, así como principales retos a futuro.

\section{La biología a la luz de la ciencia y tecnología}

En cuanto a los aspectos orgánicos de la naturaleza humana, resulta imperativo rescatar el impacto de este paradigma bajo las dimensiones propias de la Ciencia y tecnología. Por ende, disciplinas tales como: las Neurociencias, la Inteligencia Artificial, la Nanotecnología, la Bioingeniería y la Genética permitirán plantear, dentro de los corolarios éticos, el mejoramiento exponencial de la naturaleza humana como una postura presente para las futuras generaciones. Respecto a las neurociencias, autores como Cumpa-Valencia (2019) la definen como un "conjunto de saberes orientados al estudio de la estructura y funcionamiento del sistema nervioso, así como la interacción de sus componentes que dan origen a la conducta de los seres vivos". Es decir, el componente neural de los organismos adquiere un carácter interdisciplinario y transdisciplinario. Razón por la cual, uno de sus principales proyectos: el conectoma humano, establece la relevancia de tres aspectos centrales al describir las redes de elementos y conexión que realiza el cerebro humano (Sporns, 2011). Uno de aquellos aspectos abarca a la conectividad funcional de las estructuras corticales, otro alude a la descripción de bases de datos cerebrales, mientras que el último concierne a la interacción mediante redes por parte de los dos primeros. A raíz de ello, la Inteligencia artificial brinda una oportunidad amplia en el desarrollo de tecnologías exponenciales que sirvan para crear soluciones prácticas capaces de innovar el contexto virtual humano. Esto se debe a que representa "la capacidad de un sistema para interpretar datos externos correctamente, aprender de tales datos y utilizar esos aprendizajes para lograr objetivos y tareas específicas de manera adaptativa". (Haenlein \& Kaplan, 2019).
Orientado a trabajar ante un gran cúmulo de datos, existen estrategias capaces de optimizar el proceso de aprendizaje de un sistema informático mediante el uso de procesos naturales aplicados a entornos digitales, estas son conocidas como Computación Natural o Computación suave (Kook, 2004). El uso y aplicación de estas herramientas (Redes neuronales, Lógica difusa, Sistemas expertos, Algoritmos genéticos) trae consigo un avance exponencial en el desarrollo de materiales sintéticos u orgánicos capaces de ser programados o editados. Pongamos por caso la Nanotecnología que ofrece grandes aplicaciones en cuanto al uso de materia sintética programable en diversos contextos de la naturaleza humana y en el entorno que le rodea. Definida por Serrano y Seeman (2004) como "la habilidad de trabajar a nivel molecular (...) estructuras [atómicas] o dispositivos a nivel nanométrico que posean propiedades físicas, químicas o biológicas nuevas o mejoradas para ciertos fines superiores a lo que actualmente conocemos". Frente a esta concepción, la Claytrónica o materia programable, se presenta como uno de los avances aplicables a futuro. Es decir, la realidad sintética en torno a robots reconfigurables a escalas nanométricas (cátomos) es un avance científico-tecnológico capaz de amplificar las esferas propias de la naturaleza humana (Abhishekh et al., 2013; Guin, 2012).

En cuanto a la edición o programación de la materia orgánica, tanto la Bioingeniería como la Genética plantean un gran abanico de aplicaciones en diversos campos de estudio. El primero presenta a las tecnologías exponenciales como entidades capaces de optimizar y/o mejorar el rendimiento de funciones orgánicas mediante extensiones tecnológicas biocompatibles. Esto es claro en los Exoesqueletos, la Ingeniería de tejidos, las Neurotecnologías $u$ otras manifestaciones biotecnológicas cuyo objetivo implica "establecer un nuevo canal de comunicación del individuo con su medio que no dependa del accionar de vías orgánicas [o incluso potenciar el rendimiento de estos organismos]" (Gentiletti et al., 2007). En otras palabras, lo que se busca, aparte de potencializar la condición orgánica humana, es plantear futuros indicadores éticos en el desarrollo y regulación de tecnologías duales. De modo similar, la genética, se encarga de estudiar "la herencia y la variabilidad dentro de las especies" (Viedma, 2002). En este caso, el Proyecto Genoma Humano (PGH) trajo consigo el desarrollo de nuevos avances en la edición de la materia orgánica. Técni- 
cas como el Clustered regularly interspaced short palindromic repeats (CRISPR) ahora ponen sobre la mesa de trabajo no solo las implicancias bioéticas del uso y manipulación de los genes, sino también la observación de "si el derecho podrá adecuarse a la vertiginosidad de los avances tecnológicos en la materia, o si terminará claudicando ante ellos" (Cárdenas, 2019). Razón por la cual, la Organización de las Naciones Unidas para la Educación, la Ciencia y la Cultura (UNESCO) en su Declaración Universal sobre el Genoma Humano y los Derechos Humanos, trata las implicancias axiológicas de esta variable. En ella, se abordan conceptos acerca de la dignidad humana y el genoma humano, el contexto legal de las partes interesadas, así como investigaciones propias del objeto de estudio en cuestión. Asimismo, ofrece condiciones en el accionar científico, seguida de un llamado a la solidaridad y cooperación; así comoel fomento y aplicación de lo estipulado en aquel documento (Bergel, 1998). Vale decir, que esta propuesta es un indicador clave en cuanto al valor de labioética dentro del campo de la ingeniera genética. Si bien "la ciencia nos da los medios, son los valores humanos los que deben proponer los fines" (Unión Internacional Humanista y Ética [IHEU], 2002). Así pues, estos avances científicos y tecnológicos en las tecnologías NBIC, aparte de beneficiar de manera exponencial diversos sectores del desarrollo humano, también presentan determinados criterios éticos que merecen ser considerados.

\section{La psicología del ciberespacio}

Acápite aparte, dentro de la esfera Psicológica de la naturaleza humana, el desarrollo de la industria 4.0 plantea un mayor impacto, tanto en el marco de las ciencias cognitivas como en el análisis experimental de la conducta humana. Esto trajo consigo la concepción de la Ciberpsicología como "una rama reciente de la psicología cuyos principales objetos de investigación son los procesos de cambio [cognoscitivos, volitivos, conductuales] inducidos por las nuevas tecnologías" (Parsons, 2017). Es decir, las interacciones de un organismo frente a un aspecto particular del entorno virtual suelen implicar un impacto en la conducta humana y en sus diversas manifestaciones contextuales. Sumado a ello, autores como Lara y Hermosillo (2011) plantean que la aplicación de estas tecnologías en el marco cognitivo involucra grandes avances en el desarrollo de sistemas artificiales capaces de emular diversas manifestaciones cognitivas. En consecuencia, existen diferentes maneras de abordar el estudio de la cognición, que según la perspectiva de Restrepo (2018) pueden ser corporeizadas, situadas o extendidas. Ante esto, el principal reto involucra generar algoritmos capaces de obtener información de diversos entornos e interactuar con ellos de manera eficaz y eficiente a fin de crear realidades digitales propias de la industria 4.0 capaces de acelerar el auge de fenómenos convergentes tales como la robótica cognitiva, la realidad virtual, la Ciberpsicología basada en el cerebro, los sistemas de neuroinformación (NeurolS) entre otros.

Asimismo, en las interacciones socioafectivas dentro del contexto virtual, este paradigma ofrece una nueva forma de comunicar y percibir la información afectiva. En este rubro, las Ciberrelaciones se demarcan como un espacio donde "Se han desarrollado nuevas teorías para explicar cómo las personas inician, desarrollan y mantienen relaciones en el ciberespacio y cómo esas relaciones progresan hacia espacios fuera de línea" (Whitty \& Young, 2017). En términos sencillos, la expresión y percepción del afecto en las relaciones de pareja, al ser concertada en espacios virtuales, trae consigo la manifestación de la sexualidad en sus diversas expresiones. Siendo ejemplos de ello el cibersexo, elsexting1, el uso de robots sexuales e incluso la manifestación de la autorepresentación del yo durante la consecución de citas online.

Respecto al marco conductual, el avance de la Cuarta Revolución Industrial, permite un mayor nivel de graduación y registro de las tasas de respuesta por parte de los sujetos ante ciertos estímulos preestablecidos. Esto se nota en el desarrollo y aplicación de algoritmos informáticos, Bias o Nudges, en la regulación de conductas asistidas mediante un ordenador. Como resultado, investigadores como Attrill \& Fullwood (2016) refieren que las intenciones del comportamiento de las personas, hacia un determinado sitio web,tienden a vincularse con las actitudes que se manifiestan como motivadores o estímulos discriminativos de aquella respuesta. Si bien los alcances de la 4RI permiten un impacto relevante en el desarrollo de la psique humana en sus planos cognitivos, volitivos y procedimentales, es menester señalar que uno de los posibles retos a considerar es el aumento exponencial y acumulativo en la tasa de respuesta de los sujetos ante aquellas variables mencionadas anteriormente. Es decir, la concepción de conductas adictivas no bioquímicas, así como la ampliación comunicativa de sesgos y falacias cognitivas, son aspectos a considerar en el futuro capital humano. 


\section{La virtualidad de la esfera social}

En cuanto a las variables sociales inherentes a este fenómeno industrial es relevante indicar que la sociedad a lo largo del tiempo ha atravesado por diversos paradigmas propios del contexto en donde acontecen. A partir de ello, el desarrollo de las vertientes evolutivas, humanísticas e históricoculturales ofrecen un amplio panorama de los procesos interactivos, dinámicos y retroalimentativos que atraviesan sus pobladores frente al entorno propio de su tiempo. Desde el punto de vista de la hominización, definida como "la progresiva adquisición de unas características determinadas que han acabado diferenciando al ser humano del resto de los animales" (Lizaso et al., 2017). Es necesario señalar que este proceso evolutivo aun no es del todo concluyente en la naturaleza humana. Por ende, el ser humano, en la medida en que interactúe con los avances científicos y tecnológicos de su entorno, tenderá al manejo experto y diestro en el diseño de su propia estructura orgánica, tanto a nivel biológico como cognitivo. Esto se debe a que la tesis expuesta por Waterworth \& Riva (2014) alude que estos procesos han sido diseñados para ser útiles en la supervivencia y reproducción en el entorno en donde el ser humano se desarrolla. Desde esa perspectiva, resulta factible explicar una amplia gama de factores comportamentales y afianzar un nuevo tipo de explicación filosófica en cuanto a la comprensión de la naturaleza humana. De esta manera, en el campo de la humanización, el auge exponencial de los avances tecnológicos permite establecer nuevas posturas acerca del sentido de autorealización humana. Es decir, el Transhumanismo se plantea como paradigma propio del estilo de pensamiento acorde al avance, impacto y auge de la Cuarta Revolución Industrial. Si bien esta corriente "propone el Mejoramiento Humano (Human Enhancement) como medio para lograr un estado evolutivo más fructífero del Homo Sapiens" (Gayozzo, 2019), autores como Bostrom (2019) afirman la necesidad de realizar repertorios axiológicos basados en valores que hagan de la existencia posthumana un periodo digno y acorde a las metas e ideales enfocados en el bien común. Asimismo, dentro del contexto histórico-cultural, el desarrollo de los gobiernos y sus políticas públicas requieren una reconcepción del quehacer del aparato estatal frente a sus ciudadanos. De esta manera, la Extrapolítica se erige como una vertiente gubernamental orientada al establecimiento de una ciudadanía acorde a los avances tecnológicos y cien- tíficos. Para ello resulta óptimo considerar que "la política evoluciona y que es obra del Homo Sapiens" (Gayozzo, 2018). En consecuencia, la concepción de un Estado Post-Democrático necesita que los ciudadanos mantengan su voz, en aras de la ciencia, para un mundo mejor. Aspecto que no estaría sujeto a una labor de elecciones verticales centralizadas en un cargo, sino al contrario, que permita la consecución de "una sociedad que realmente se construya a sí misma gracias a la democratización de espacios de trabajo e ideación de proyectos (...) que fomenten el autogobierno concienzudo" (Gayozzo, 2020). No obstante, al contar con un mayor acceso a la información y gestión del conocimiento en plataformas digitales, resulta imperativo considerar los posibles retos a futuro. Frente a esta observación, fenómenos colectivos como la polarización en redes sociales y la cultura de la cancelación se vuelven indicadores de rechazo social dentro del ciberactivismo. Agregado a ello, la narrativa semiótica, en materia de contenidos impartidos por influencers1 y sus tendencias en redes sociales, son activos capaces de marcar la pauta de una futura agenda Extrapolítica al momento de construir un estado postdemocrático.

\section{El contexto bajo interacciones en línea}

Por último, otro de los factores que se verán inmersos en el modelo industrial 4.0 son aquellos relacionados con el contexto donde se producen las interacciones humanadas. Instituciones como la familia, la escuela, las corporaciones e incluso el ambiente, tenderán a ser entidades dinámicas y dialécticas, al momento de afrontar este paradigma emergente y exponencial. En el campo de las familias, el impacto de la Industria 4.0 ha de permitir un mayor auge en la comunicación e interacción familiar. Esto se debe a que la tecnología permite la construcción de vínculos filiales entre los miembros de un sistema parental. En cuanto a las consideraciones sobre una relación dinámica entre sus integrantes, autores como Barrera \& Duque (2014) refieren que "según el nivel de manejo de internet que tienen los padres de familia, se establecen particulares tipos de interacción con sus hijos". Es decir, la parentalidad en línea requiere ajustarse no sólo en capacidades de alfabetización tecnológica, sino también en la búsqueda de la perpetuidad de comunicación e interacción entre los familiares. A fin de estar atentos ante los posibles riesgos en que se hallan inmersos los jóvenes internautas, tales como el ciberbullyng, la suplantación de identidad, el acceso no autorizado a 
datos privados, entre otros. De manera similar, en el ámbito educativo, las tecnologías propias del 4RI servirán como una herramienta eficaz y eficiente en el manejo de los retos educativos del aprendiz. A nivel metodológico, los Sistemas tutoriales inteligentes (STI) recortarían las brechas entre la sistematización del contenido curricular y la exposición del aprendiz a estos conocimientos al grado de considerar, dentro del análisis exhaustivo del sistema, el ritmo de aprendizaje y nivel de desempeño metacogintivo que manifieste el alumno mientras construye su propio conocimiento. Esto se debe a que son "Sistemas que se adaptan al estado de conocimiento del alumno y al estilo de enseñanza del tutor cuya efectividad radica en la enseñanza uno-a-uno" (Huapaya et al., 2005). En otros términos, el campo metodológico dentro del rol docente sería el principal beneficiario en la implementación de estos sistemas. Como resultado, "el acompañamiento personalizado podría ser mucho más efectivo y las decisiones de asesoría académica específica se realizarían en base a datos concretos" (Crovetto, 2020). Aunado a ello, dentro del marco didáctico, el uso de estrategias como el STEAM (Science, Technology, Engineering, Arts, Mathematics) ha permitido exponer y vislumbrar un gran paso en cuanto a la aplicación de contenidos científico-tecnológicos en la enseñanza de las escuelas. Razón por la cual, este enfoque "es clave para preparar a la nueva generación en los retos que nos depara nuestro mundo de caudales tecnocientíficos y los desafíos que tenemos como país" (Mori, 2020). En consecuencia, el impacto de la Cuarta Revolución Industrial en el sector educativo trae consigo una serie de cambios vertiginosos que vale la pena considerar, si lo que se busca es optimizar de manera eficaz y eficiente la calidad educativa a través de sus aportes. En ese sentido, la esfera laboral del paradigma 4RI, genera un replanteamiento del proceso de manejo de los recursos y el capital humano en el campo de las organizaciones.

A nivel tributario, el desarrollo de estrategias como el Blockchain, el Inteligence Bussinnes, el Legal tech o la Artificial Intelligence en procesos de selección de personal, mantienen un rol importante en el marco organizacional de las industrias 4.0. Incluso, el desarrollo de la Hiperautomatización permite establecer un indicador de valor agregado al momento de combinar el aprendizaje automático y los paquetes informáticos. Esto quiere decir que "virtualmente todas las tendencias tendrán un im- pacto en personas y espacios inteligentes" (Meza, 2020). Argumento por la cual, el aporte de los avances tecnológicos y científicos de la Cuarta Revolución Industrial requiere una reincorporación funcional en las áreas de Investigación, Desarrollo e Innovación $(I+D+i)$ en cada uno de los ámbitos organizacionales. Una tarea pendiente que, en caso de concretarse, permitirá optimizar la toma de decisiones en la gestión y alta dirección de las empresas.

Algo similar ocurre en la gestión ambiental, donde la 4RI ofrece replanteamientos en la preservación y conservación de la biodiversidad. La reducción de emisiones de gases invernaderos, la exploración de nuevos entornos extraplanetarios, la creación de fuentes de energía exógenas e incluso, la conservación del material genético de especies vulnerables o extintas(Abely et al., 2019) soninteresantes avances en la sostenibilidad medioambiental. Sin embargo, un aspecto a considerar también son las implicancias en cuanto al concepto de industrias 4.0 responsables con el medio ambiente. Razón por la cual, Vázquez et al. (2020) establecen una revisión del Informe de Brundtlan de 1987, cuya vigencia epistemológica lleva con nosotros 33 años. A fin de poner sobre la mesa, una precisión del término Sostenibilidad ecológica por uno de Sostenibilidad tecnológica para que, de esta manera, se continúe con la labor de conservación de recursos naturales bajo un marco epistemológico acorde a los avances tecnológicos y científicos de su época. En fin, aunque el contexto familiar, educativo, laboral y ambiental se modifiquen por el impacto de la Industria 4.0, vale la pena observar en detalle la necesidad de establecer un contexto más integrador dentro de un marco de políticas públicas orientadas al bienestar común acerca de los futuros retos en cada uno de ellos. Caso contrario, las brechas presentes, han de acrecentar la percepción de la población ante una realidad del tipo High tech-Low life, típico de obras del género Cyberpunk7. Una tarea pendiente de la cual todos y cada uno de nosotros hemos de formar parte, ya que, la realidad de la humanidad, es la narrativa que se asume dentro de un colectivo.

\section{Palabras finales}

En conclusión, la Cuarta Revolución Industrial trae consigo nuevas áreas de desarrollo de procedimientos científico-tecnológicos orientados al beneficio de la humanidad. Aspectos en los cuales las tecnologías NBIC permitirán superar las limitaciones biológicas de la naturaleza humana. De 
manera que la psicología del futuro ciudadano inmerso en este contexto se modifique a nivel cognitivo, volitivo y conductual. Así pues, el Transhumanismo, desde su perspectiva crítica, permitirá precisar, tanto las implicaciones éticas como las futuras estructuras sociales que consignen en el desarrollo histórico-cultural del ser humano. Una realidad emergente, cuyas interacciones son capaces de modificar el contexto familiar, educativo, laboral y ambiental de la humanidad. Al fin y al cabo, con esta iniciativa, se espera sentar las futuras bases para un posterior diálogo acerca del impacto y los retos de la Industria 4.0 en aquellos hitos relevantes para el desarrollo económico de la sociedad, tales como: la salud, la educación, la ciencia y la tecnología. Ya que como versa la siguiente afirmación: Con ciencia, hay futuro, y si es con ética, el provenir suele ser mucho mejor.

\section{Referencias}

Abely, T., Dalrymple S., Godefroid S., Mondoni A., Muller J., Rossi G. \& Orsenigo S. (2019). Ex situ collections and their potential for the restoration of extinct plants. Conservation Biology, 34(2), 1-11. https://doi.org/10.1111/cobi.13391

Abhishekh, D., Ramakantha, B., Vijaya, Y. \& Basi, A. (2013). Claytronics - A synthetic reality. International Journal of Scientific and Engineering Research, 4(3), 1-4. https://www.ijser.org/researchpaper/Claytronics-A-Synthetic-

\section{Reality.pdf}

Attrill A. \& Fullwood C. (2016). Applied Cyberpsychology Practical applications of cyberpsychological theory and research. Palgrave Macmillan.

Barrera,D. \& Duque, L. (2014). Familia e internet: consideraciones sobre una relación dinámica. Revista Virtual Universidad Católica del Norte, (41), 30-44. https://www.redalyc.org/articulo.oa? id=194229980004

Bergel, S. (1998). La declaración universal de la UNESCO sobre el genoma humano y los derechos humanos. Cuadernos de Bioética, 9(34), 387-405. http://aebioetica.org/revistas/1998/2/34/387.pdf

Bostrom, N. (2019). Valores transhumanistas. Instituto de Extrapolítica y Transhumanismo. https://extrapolitica.ssh.org.pe/wpcontent/uploads/2020/02/Bostrom-Nick-Valores-TranshumanistasInstituto-de-Extrapol\%C3\%ADtica-y-Transhumanismo.pdf Cárdenas, R. (2019). CRISPR y el nacimiento en China de dos niñas editadas genéticamente. Instituto de Extrapolítica y Transhumanismo.

Crovetto, A. (2020). ¿Quieres que te enseñe SIRI?: Inteligencia artificial en la educación.Instituto de Extrapolítica y Transhumanismo. https://doi.org/10.52749/iet.v3i1.15

Cumpa-Valencia, M. (2019). Usos y abusos del término "neurociencias": Una revisión sistemática en revistas indexadas Scielo. Revista ConCiencia EPG, 4(1), 30-67. https://dialnet.unirioja.es/servlet/articulo?codigo $=7304977$

Gayozzo, P. (2020). El Estado Post-Democrático. Instituto de Extrapolítica y Transhumanismo. https://doi.org/10.52749/iet.v3i1.10 Gayozzo, P. (2020). La cuarta revolución industrial: Una revolución venidera. Futuro Hoy, 1(1), 11-13. https://doi.org/10.52749/fh.v1i1.2 Gayozzo, P. (2019). ¿Qué es el transhumanismo?. Instituto de Extrapolítica y Transhumanismo. https://doi.org/10.52749/iet.v2i1.9 Gayozzo, P. (2018). Teoría Extrapolítica y Postpoliticismo: Sobre la Vía de la Política, la evolución del hombre y la singularidad e inflexión bio-socio-política. Instituto De Estudios Transhumanistas, 1(1). https://doi.org/10.52749/iet.v1i1.6
Gentiletti G., Taberrning C. \& Acevedo R. (2007). Interfaz CerebroComputadora: Estado del arte y desarrollo en Argentina. Revista Argentina de Bioingeniería, 13(1), 22-29.

https://www.researchgate.net/publication/262568028_Interfaz_Cerebr o_-Computadora_Estado_del_arte_y_desarrollo_en_Argentina Guin, A. (junio, 2012). Programmable matter- Claytronics

[Presentacion de paper]. 58th International Instrumentation Symposium, California, USA.

https://www.researchgate.net/publication/305318988_Programmable _Matter_-_Claytronics

Haenlein, M. \& Kaplan, A. (2019). A brief history of Artificial Intelligence: On the past, present and future of Artificial Intelligence. California Management Review, 61(4), 1-10.

https://doi.org/10.1177/0008125619864925

Huapaya, C., Arona, G. \& Lizarraide F. (2005). Enseñanza de la ingeniería con Sistemas Tutoriales Inteligentes. Información Tecnológica, 16(5), 75-78. http://dx.doi.org/10.4067/S071807642005000500012

Kook,W. (2004). Análisis del estado del arte en Algoritmos genéticos [Tesis de grado, Universidad de los Andes]. Séneca Respositorio instirucional. https://repositorio.uniandes.edu.co/handle/1992/21408? locale-attribute $=$ en

Lara, B. \& Hermosillo, J. (2011). Inteligencia y robótica corporizada. https://es.scribd.com/document/472728354/235027468-Inteligenciay-Robotica-Corporizada-pdf

Lizaso I., Acha J. , Reizabal L . \& García A. (2017). Desarrollo biológico y cognitivo en el ciclo vital. Ediciones Pirámide.

Meza, D. (2020). Hiperautomatización, un reto hacia el 2030. Futuro Hoy, 1(1), 33-34. https://futurohoy.ssh.org.pe/wpcontent/uploads/2020/12/Meza-Daniel-2020.-

Hiperautomatizaci\%C3\%B3n-un-reto-hacia-el-2030.-Futuro-Hoy.Vol.1-Nro.1.pdf

Mori, A. (2020). El reto educativo del siglo XXI: el enfoque STEAM en la Cuarta Revolución Industrial. Futuro Hoy, 1(1), 19-21.

https://doi.org/10.52749/fh.v1i1.5

Parsons T. (2017). Cyberpsychology and the brain. Cambrigde University Press.

Restrepo, J. (2018). Cognición corporizada, situada y extendida: una revisión sistemática. Revista Katharsis, 26, 106-130.

https://dialnet.unirioja.es/servlet/articulo?codigo $=6796566$

Schwab K. (2016). La cuarta revolución industrial. El Tiempo Casa Editorial, S. A. 
Sporns, O. (2011). The human connectome: A complex network. Annals of the New York academy of sciences, 1224(1), 109-125. https://www.researchgate.net/publication/49770658_The_human_con nectome A complex network

Torró, LI. (1999). La revolución industrial. https://www.researchgate.net/publication/39383584_La_revolucion_i ndustrial

Unión Internacional Humanista y Ética. (2002). Declaración de Ámsterdam. Revista Latinoamericana de Psicología, 35(2), 235-236. https://www.redalyc.org/articulo.oa?id=80535221

Vázquez, A., Cabrera I. \& Portuondo R. (2020). ¿Sostenibilidad ecológica o sostenibilidad tecnológica? Monteverdia, 13(1), 67-75. https://revistas.reduc.edu.cu/index.php/monteverdia/article/view/3610 /3301
Viedma, I. (2002). Proyecto genoma humano Implicaciones éticas. Persona y Bioética, 6(17), 73-84.

https://personaybioetica.unisabana.edu.co/index.php/personaybioetic a/article/view/847/929

Waterworth J. \& Riva G. (2014). Feeling Present in the Physical World and in Computer-Mediated Environments. Palgrave Macmillan. Whitty M. \& Young G. (2017). Cyberpsychology:The study of individuals, society and digital technologies. The British Psychological Society and John Wiley y Sons, Ltd.

\section{Cómo citar este artículo:}

Siancas, W. (2021). La Cuarta Revolución Industrial: un paradigma emergente en la sociedad de hoy. Futuro Hoy, 2(4), 17-23. https://doi.org/10.52749/fh.v2i4.3

Esta obra está bajo licencia internacional

Creative Commons 4.0 Reconocimiento 4.0. 